\title{
Hand Radiograph Analysis and Joint Space Location Improvement for Image Interpretation
}

\author{
BARTOSZ ZiELIŃSKI \\ Faculty of Physics, Astronomy and Applied Computer Science \\ Jagiellonian University, Reymonta 4, 30-059 Kraków, Poland \\ e-mail: bartosz.zielinski@uj.edu.pl
}

\begin{abstract}
Hand radiograph analysis is extremely exhausting and time consuming for radiologists, not because of the complexity, but because of the precision required during diagnosis. Due to this fact, automatic analysis of the joint space width of all joints in a hand would be indispensable. In the following paper, an improvement of a method used to quantify the minimal JSW in hand radiographs, proposed by the author in his previous works, is considered. The proposed changes have an impact on initial pre-processing and joint space location. As a result of experiments conducted on more than 1100 joint spaces, the overall error of pre-processing and joint location decreases from $10.8 \%$ to $2.59 \%$ after changes.
\end{abstract}

Keywords: image processing, medical imaging, radiology, rheumatoid arthritis, X-ray photo, thresholding, joint space location, joint space width.

\section{Introduction}

Arthritis and musculoskeletal disorders are more prevalent and frequent causes of disability than heart disease or cancer [11]. There are a number of inflammatory as well as non-inflammatory diseases within the scope 
of rheumatology and diagnostic radiology. It is essential to distinguish between inflammatory disorders, which can be fatal, and non-inflammatory, which are relatively harmless and can afflict majority of people aged around 65. Rheumatoid arthritis is a model of an inflammatory disease that affects $0.5-1 \%$ of the population worldwide [12]. Osteoarthritis is a model of a noninflammatory disease and is the most common joint disorder in the world [7].

To give a diagnosis, an X-ray is taken of the patients hand and symmetric metacarpophalangeal joint spaces and interphalangeal joint spaces are analyzed [20]. Due to the number of hand joints, regular analysis is exceedingly complicated and rarely carried out by specialists. Consequently, a diagnosis is usually made only when the pathological changes are significant. However, it is extremely important to diagnose pathological changes in the early stages of a disease, which means that differences in the order of $0.1 \mathrm{~mm}$ between the contours of pathologically changed bones and unaffected ones need to be identified.

To make X-ray examinations more frequent and more precise, the process has been automated. However, computer studies of hand X-ray pictures are extremely difficult because, amongst other things, complex bone spatial relations include mutual coverings which can overlap or cover each other in an X-ray picture. Thus, until now, there have been only a few pioneer papers concerning this topic $[2,3,4,10,21,22,16,17,19,23]$.

The aim of the conducted research is to create an X-ray based expert system aiding diagnosis of hand diseases. The software designed and implemented so far (see [2]) pre-processes an X-ray image and returns a set of minimal joint space widths in five steps - initial binarisation, thinning, branch analysis, joint space location, identifying the borders of the upper and lower surfaces and finding minimal joint space widths.

In this paper, an improvement of initial binarisation and joint space location methods are described. Modified algorithm returns correct location of joints more frequently than before changes.

\section{Some data concerning the subject of our investigation}

Routine, conventional, hand and wrist radiographs are a source of important clinical information with regard to very prevalent musculoskeletal diseases [1]. Radiographs are used not only for initial diagnosis but also for the monitoring of a diseases progression and assessment of the therapeutic effect of various drugs [15]. 


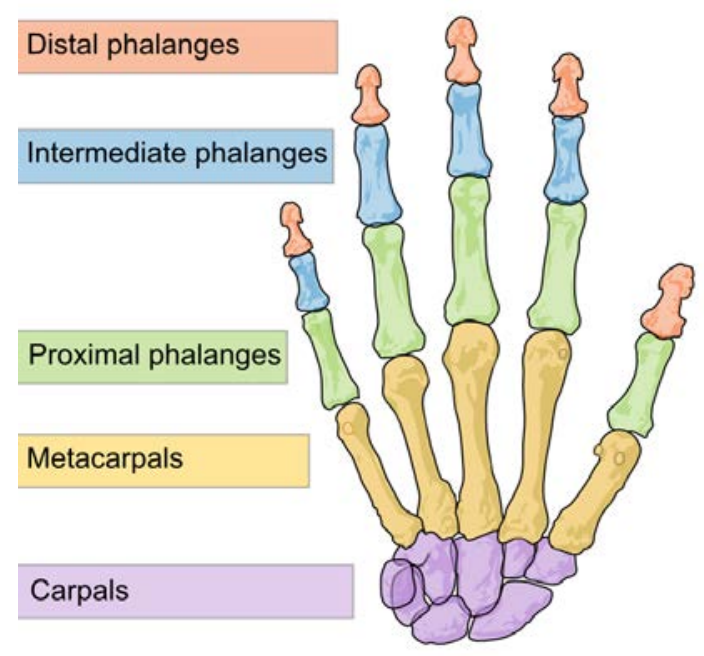

Fig. 1. Hand anatomy

In general there are two groups of rheumatic musculoskeletal diseases. The first group is described as inflammatory disorders, with rheumatoid arthritis being the most prevalent and used in our research as a model of an inflammatory disease. The second group is known as non-inflammatory disorders which comprise degenerative diseases of the joints, i.e. osteoarthritis, the most prevalent and the one used in our research as a model of noninflammatory diseases.

One of the most significant radiological features of non-inflammatory and inflammatory disease is joint space narrowing (JSN) in the symmetrical joints of the left and right hand. As a consequence of our analysis process, the joints with pathological changes (Fig. 2b) should be educed from healthy joints (Fig. 2a).

There are three kind of joint considering hand pathology in rheumatoid arthritis and osteoarthritis (Fig. 1). The first is the metacarpophalangeal joints (MCP joints between the metacarpal bones and proximal phalanxes). The proximal interphalangeal joints (PIP joints between the proximal and middle phalanxes) are the second region of interest whereas the third one are the distal interphalangeal joints (DIP joints between the middle and distal phalanxes). There are neither PIP nor DIP joints in the first finger due to there being only two phalanxes, proximal and distal, and the only joint between the phalanxes is called the interphalangeal (IP) joint.

The important and indicative features are distribution (MCP, PIP, DIP joints) and symmetry (both sides) of any changes. In rheumatoid arthritis, 


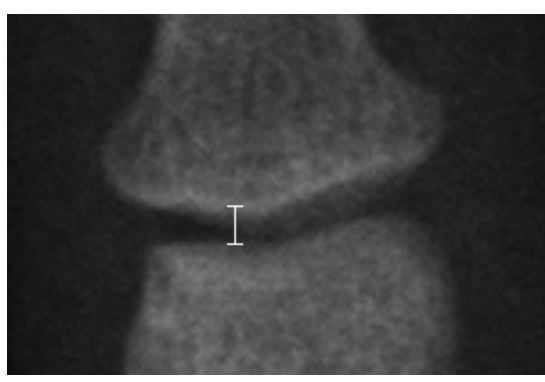

a)

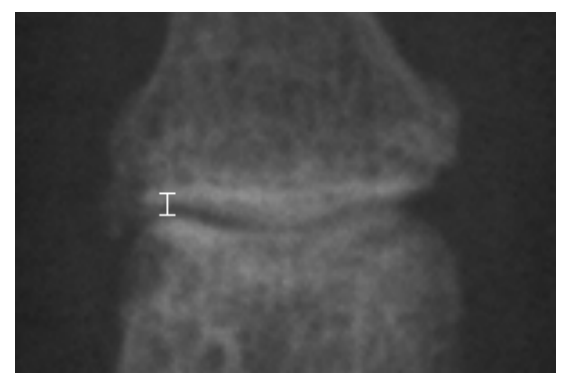

b)

Fig. 2. Joint width within normal limits - approximately $2 \mathrm{~mm}$ (a), and a narrowed joint - less than $1 \mathrm{~mm}$ (b)

joint space narrowing is seen exclusively in MCP and PIP joints and changes are symmetric (in both hands and all joints); in osteoarthritis only PIP and DIP joints are involved, changes are asymmetric and they could spare several joints. Thus, assessing joint space width in a diagnostic process requires taking into account the distribution of narrowed hand joints (MCP, PIP or DIP) and their symmetry.

Our research which focuses on automatic assessment of joints space could facilitate radiologic measurement even once every three months which is much better than routinely every $6-12$ months. At present, the 3 month interval is a real challenge, even for the very experienced radiologist, but it speeds up significantly the decision whether to start or change the therapy if such action is feasible.

\section{State of the art}

Because of the complexity of hand construction, hand X-ray segmentation methods published so far either did not provide satisfying results [3], were not fully automated $[4,21,22,16,17,19]$ or did not analyze all joints [10].

It is particularly noticeable in Lehmann's method [3], based on watershed and rule-based merging, which results in $84.3 \%$ correct segmentations. However, the results do not guarantee gaining all joint locations from the image, especially when the analysis is conducted on older people's X-ray photos. Segments obtained with such an algorithm often meld into each other, making joints undetectable. 
Traditional methods of segmentation, like Edge Detection, Top Hat or Black Top Hat did not return satisfactory results either [3].

Another possibility was to introduce star section algorithms for segmentation $[5,6]$. Although this technique has already been applied by Tadeusiewicz and Ogiela to achieve wrist bone segmentation [21, 22], it is semi-automatic and would require a radiologist to mark 14 (respectively to the number of phalanx bones), or more central points for each X-ray photo analyzed.

Most joint space width measurement methods analyze a finger joint by detecting joint edges within a rectangular region of interest (ROI) drawn around the joint by a radiologist. This method has been applied by Pfeil et al. to estimating joint space width [16], investigating joint width differences dependent on sex and age [17] and revealing reduction of bone mineral density [4]. Similar experiments has been conducted by Sharp et al. [19] for measuring joint space widths and estimating erosion volume.

As most introduced algorithms use the ROI idea, there are only a couple of papers concerning automated joint space location. Most recent work and the actual work of van Klooster et al. [10] locates joints in $97.5 \%$ of cases using the neural network. Nevertheless, van Klooster et al. admitted location displacement of $8 \mathrm{~mm}$ from the accurate joint location, when typical joint space width oscillated between $1 \mathrm{~mm}$ and $2 \mathrm{~mm}$. Moreover, the thumb was omitted during analysis.

The method proposed by the author in his previous paper [2] not only concerns all of the fingers (including thumb), but also accepts for future analysis joint location with no displacement. However, it located joints in no more than $89.2 \%$ of cases. In this paper an improvement of the above mentioned

method is described. Applying new features increased its effectiveness and made it comparable with the one from van Klooster et al.

\section{The prior algorithm for finding joint space location}

To exploit the algorithm described in our previous article [2] and in current work, analyzed images need to satisfy the following criteria:

a) an analyzed image must be a grayscale digital image,

b) it must contain only one hand leant at a relatively small angle,

c) fingers need to be straight and cannot adjoin each other.

If all the given conditions are met, analysis is possible. 


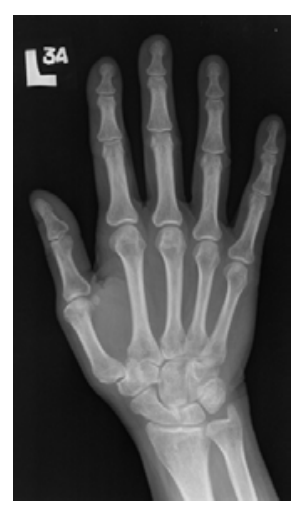

a)

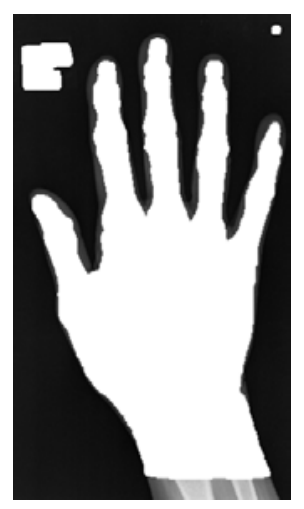

b)

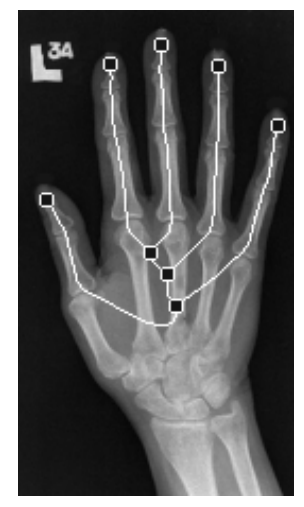

c)
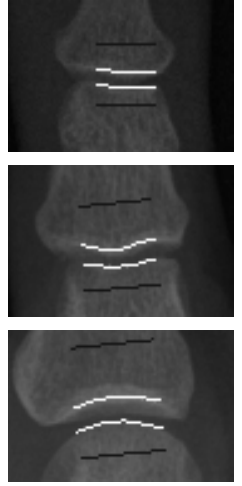

d)

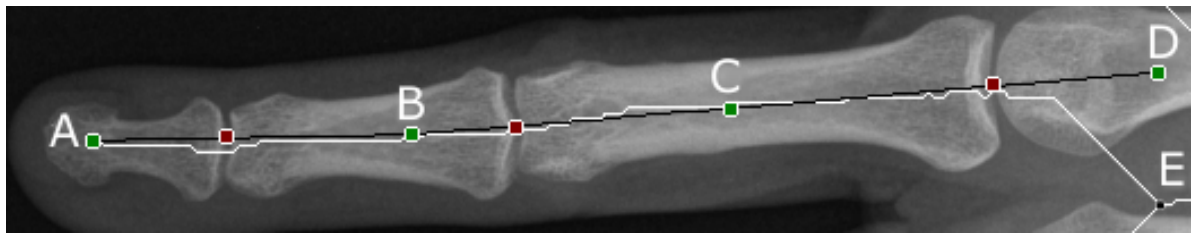

e)

Fig. 3. Exemplary input image (a), the binary image obtained as a result of the Otsu algorithm combined with an input image (b), five proper branches obtained from Fake-Branch-Elimination procedure applied to the input image (c), border of the lower and upper surface of the middle finger (d) and joint space location schema (e)

The presented algorithm is a multi-step algorithm, which means that the data obtained at each stage is used in the following stage. As an input, the algorithm requires part of an image (Fig. 3a) and as a result, the minimal joint space width for each joint is returned.

At the beginning of the analysis, the global thresholding method proposed by Otsu in 1979 [13] is used to obtain a binary image. The achieved result narrows the analyzed area to the hand pattern (see Fig. 3b). To help global thresholding receive more precise hand patterns, image pre-processing is performed before binarisation (dilation and smoothing).

In the next phase, the binary image is reduced to a one pixel thick hand skeleton (Fig. 3c) by a thinning operation [18] with an expanded set of structuring elements. In consequence, every finger possesses its own start pixel (point A in Fig. 3e) and a one pixel thick branch passing through it (white curve in Fig. 3e).

Afterwards, from all the branches obtained in the preceding stage, five proper branches are acquired. The proper branch is a branch that runs through the finger and is in a superior position to any other branch located 
in the finger. Proper branch acquisition is made by a special function: FakeBranch-Elimination [2], based on chain code [8,9] that describes the path.

Since each of the five proper branches runs through the middle of a corresponding finger, every start pixel and a proper branch are now used to compute the joint locations. Every proper branch runs through both interphalangeal joint spaces - naturally only through one in the case of the thumb. Due to this regularity, it is possible to create a profile plot utilizing the values of the pixels underneath the proper branch and use it to work out the precise location of the joint space. Before that, the scope of the profile plot has to be limited to a part named usable section and divided into three parts for analysis, as there are three joints in every finger (two in the thumb). As a result of this division, three line segments cohered to usable section, each including a joint, are created and three profile plots are analyzed to determine joint locations. The function searched for the maximal difference between the local minimum value and proceeding local maximum value.

To obtain minimal joint space width, the area of analysis of each joint is narrowed to the region of interest, using joint location point as the center of a rectangle. When the rectangle is given, many profile plots parallel to line segment are analyzed. This final action enables the gaining of the pattern of the upper and lower surface (Fig. 3d) and in consequence, reveals the minimal joint space widths (JSW).

\section{Improvement of the prior method}

Although the algorithm depicted briefly in section returned incorrect minimal JSW only in $18.4 \%$ of cases, the performance had to be improved due to the significance of precision in medical diagnosis. In the previous paper, the performance was examined by measuring the percentage error in three steps: thresholding, joint space location and calculating the minimal joint space width.

\subsection{Thresholding improvement}

In some cases of thresholding, a threshold produced a hand pattern containing holes or pieces of background, which could result in the creation of a wrong skeleton and prevent successful branch analysis. Additionally, a problem occurred when distal phalanges were eroded or the difference between 


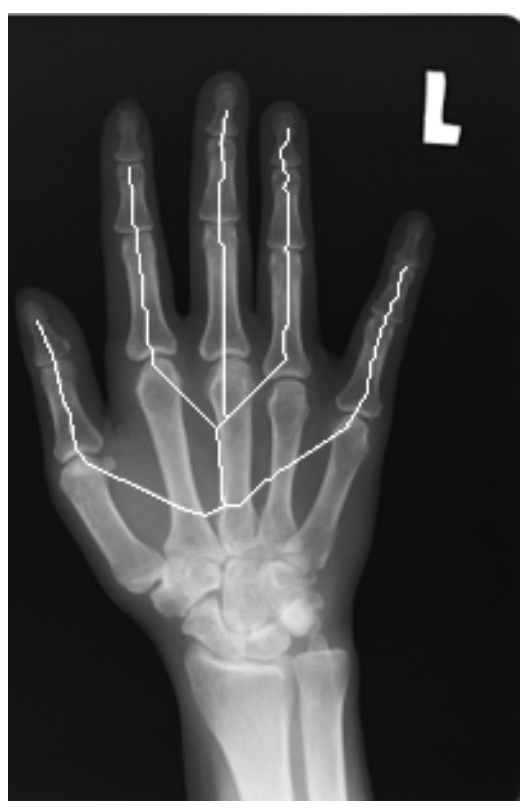

a)

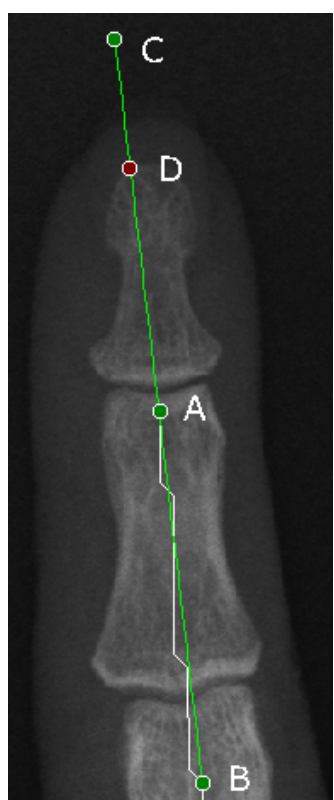

b)

Fig. 4. Skeleton with incorrect branches in index and little finger (a) and line segment of DPBP analysis (b)

background color and distal phalange color in the X-ray picture was too small. The skeleton produced from that kind of pattern contained branches which did not run trough some of the distal phalanges (see Fig. 4a).

To eliminate holes from the hand pattern, the Fill-Recursive method proposed by Pavlidis [14] was used (see Procedure 1). At first, a point placed near the border of the image was chosen as a background point. Then, the Fill-Recursive method was called with three parameters a background point (p), a hand pattern binary image (I) and a binary image with true values for each pixel $(\mathrm{J})$ - working image. When the procedure terminates, the third parameter $(\mathrm{J})$ contains a hand pattern binary image with no holes.

To neutralize the problem of incorrect branches, every start pixel and the branch is used to compute the distal phalange border point (DPBP) locations. First, line segment $B A$ is created (see Fig. 4b), where $A$ is a start pixel and $B$ is the $N$-th pixel from the branch, where $N$ is one tenth of the analyzed image height (parameter obtained experimentally). Secondly, the $C$ point and $C A$ line segment are calculated by extending the $B A$ line segment. Then, a profile plot is created, utilizing the values of the pixels underneath the $C A$ line segment (see Fig. 5). Finally, the profile plot is analyzed and DPBP are achieved with the following function. 


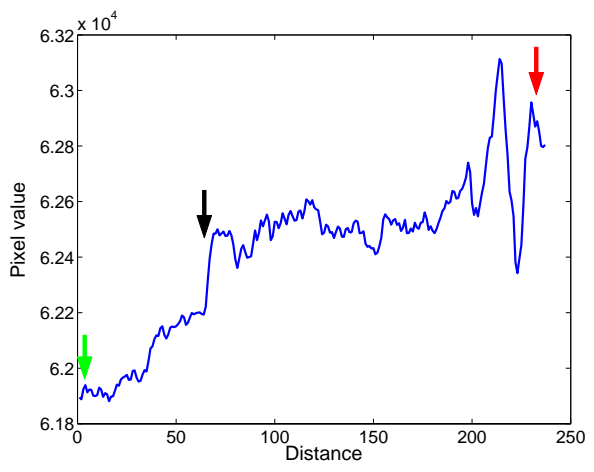

Fig. 5. The profile plot created, utilizing the values of the pixels underneath line segment $C A$ from Fig. $4 \mathrm{~b}$

Procedure 1. Algorithm for the elimination of holes from a hand pattern.

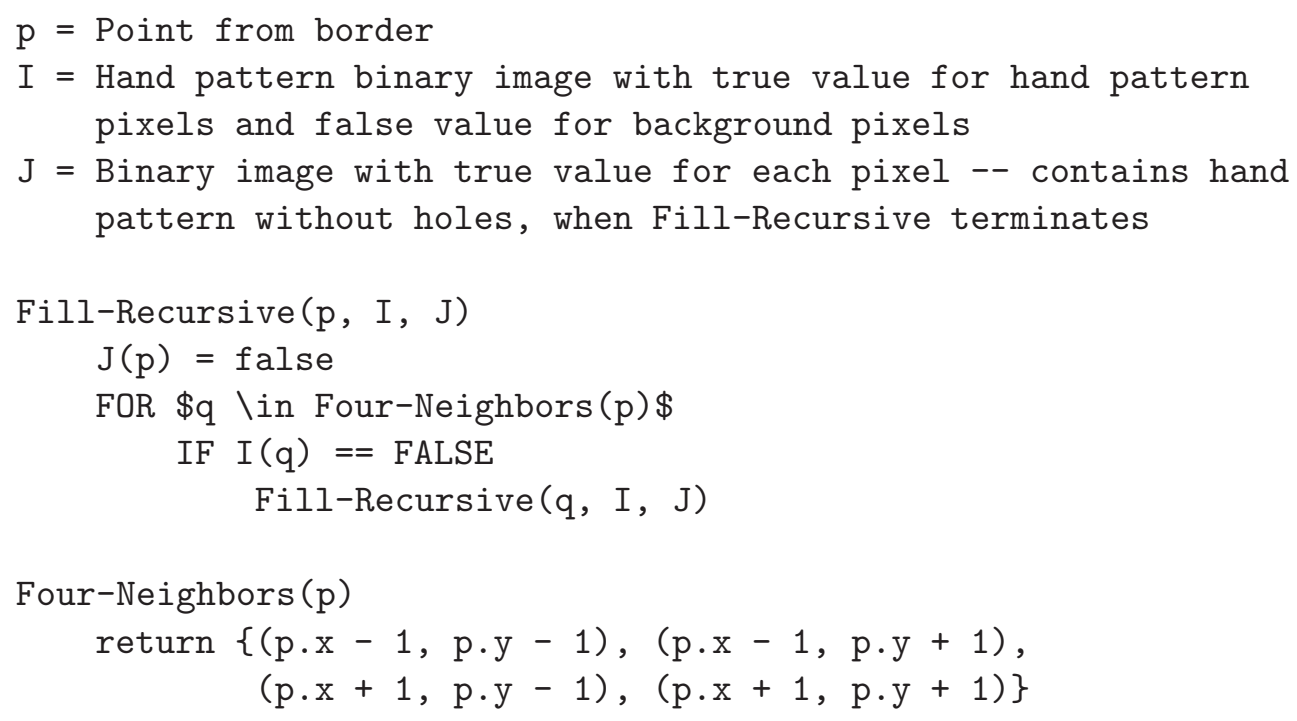


Denote by $\operatorname{avg}(n), n \in 1, \ldots, N$, average value of function $f$ in the neighbourhood of the argument $n$. The argument corresponding to the DPBP is given by the formula (middle arrow in Fig. 5):

$$
\begin{array}{r}
D P B P_{\text {argument }}:=\widehat{n}: \operatorname{avg}(\widehat{n})>\frac{\operatorname{avg}(0)+\operatorname{avg}(N)}{2} \wedge \\
\bigwedge_{n<\widehat{n}} \operatorname{avg}(n) \leq \frac{\operatorname{avg}(0)+\operatorname{avg}(N)}{2},
\end{array}
$$

where $\arg (0)$ and $\arg (N)$ are the average value of function $f$ at the beginning (first arrow in Fig. 5) and at the end (last arrow) of the profile plot, respectively.

When all DPBPs are known, each proper branch is modified. DPBP becomes a start pixel and a path from DPBP to the prior start pixel becomes part of the branch. Consequently all branches go through all phalanges, which enables future analysis.

\subsection{Improvement of joint space location}

Joint space location also produced incorrect results due to the erosion in phalanges and the small JSW. It was also discovered that profile plots created for some parts of usable areas could be more accurate when shifted left or right. These factors have a negative impact on computing the argument corresponding to joint space location given by the formula presented in the previous paper. To improve this step of the algorithm, new formula have been introduced.

The joint space location formula presented in our previous article did not take into consideration all aspects of the profile plot in arguments near arguments corresponding to a joint space location. The prior function searched for the maximal difference between the local minimum value (3rd arrow in Fig. 7) and proceeding local maximum value (2nd arrow). It proved that there are more aspects of this region. Just before local maximum (2nd arrow), there is a local minimum (1st arrow). In contrast, just after local minimum (3rd arrow), there is local maximum (4th arrow) and again local minimum (5th arrow). All of this information should be used in the new version of the formula, though with different priorities (figured experimentally). At this juncture, the improved function is defined as:

Denote by $x_{n}, n \in\{1, \ldots, N\}$, the set of those arguments for which the function $f$ reaches its local maxima. Denote by $y_{m}, m \in\{1, \ldots, M\}$, the set of those arguments for which the function $\mathrm{f}$ reaches its local minima. 


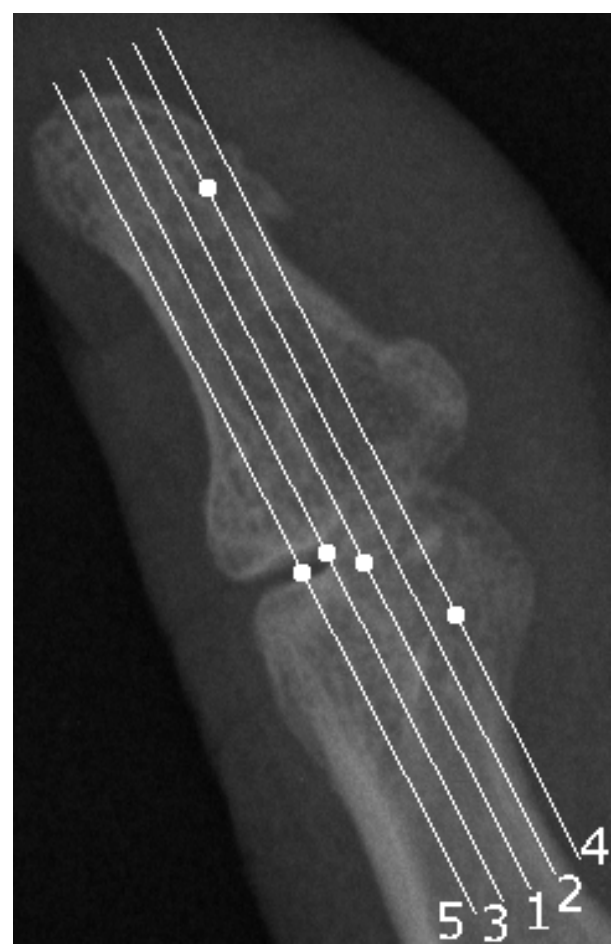

Fig. 6. Line segment (1) shifted to the left $(3,5)$ and right $(2,4)$

Let JointSpaceMaxWidth be a fixed parameter, which refers to the maximal width of a joint space, and let $\left\{\left(y_{i}, x_{j}, y_{k}, x_{l}, y_{o}\right)\right\}$ be a set of quintuples of arguments of the $\mathrm{f}$ function, so that for each $i, k, o \in\{1, \ldots, M\}$ and each $j, l \in\{1, \ldots, N\}:$

a) $y_{i}<x_{j}$ and $x_{j}-y_{i}<$ JointSpaceMaxWidth,

b) $x_{j}<y_{k}$ and $y_{k}-x_{j}<$ JointSpaceMaxWidth,

c) $y_{k}<x_{l}$ and $x_{l}-y_{k}<$ JointSpaceMaxWidth,

d) $x_{l}<y_{o}$ and $y_{o}-x_{l}<$ JointSpaceMaxWidth.

The argument corresponding to joint location point is given by the formula:

$$
\begin{array}{r}
y_{\text {joint }}:=\widehat{y}_{k}: f\left(\widehat{x}_{j}\right)+f\left(\widehat{x}_{l}\right)-2 f\left(\widehat{y}_{k}\right)+\frac{f\left(\widehat{x}_{j}\right)-f\left(\widehat{y}_{i}\right)}{4}+\frac{f\left(\widehat{x}_{l}\right)-f\left(\widehat{y}_{o}\right)}{8}= \\
\arg \max _{i, j, k, l, o}\left[f\left(x_{j}\right)+f\left(x_{l}\right)-2 f\left(y_{k}\right)+\frac{f\left(x_{j}\right)-f\left(y_{i}\right)}{4}+\frac{f\left(x_{l}\right)-f\left(y_{o}\right)}{8}\right] .
\end{array}
$$

Even though the changed formula succeeds more frequently than the previous one, it is still possible that the line segment utilized for profile plot creation goes through an eroded area and returns faulty joint space loca- 

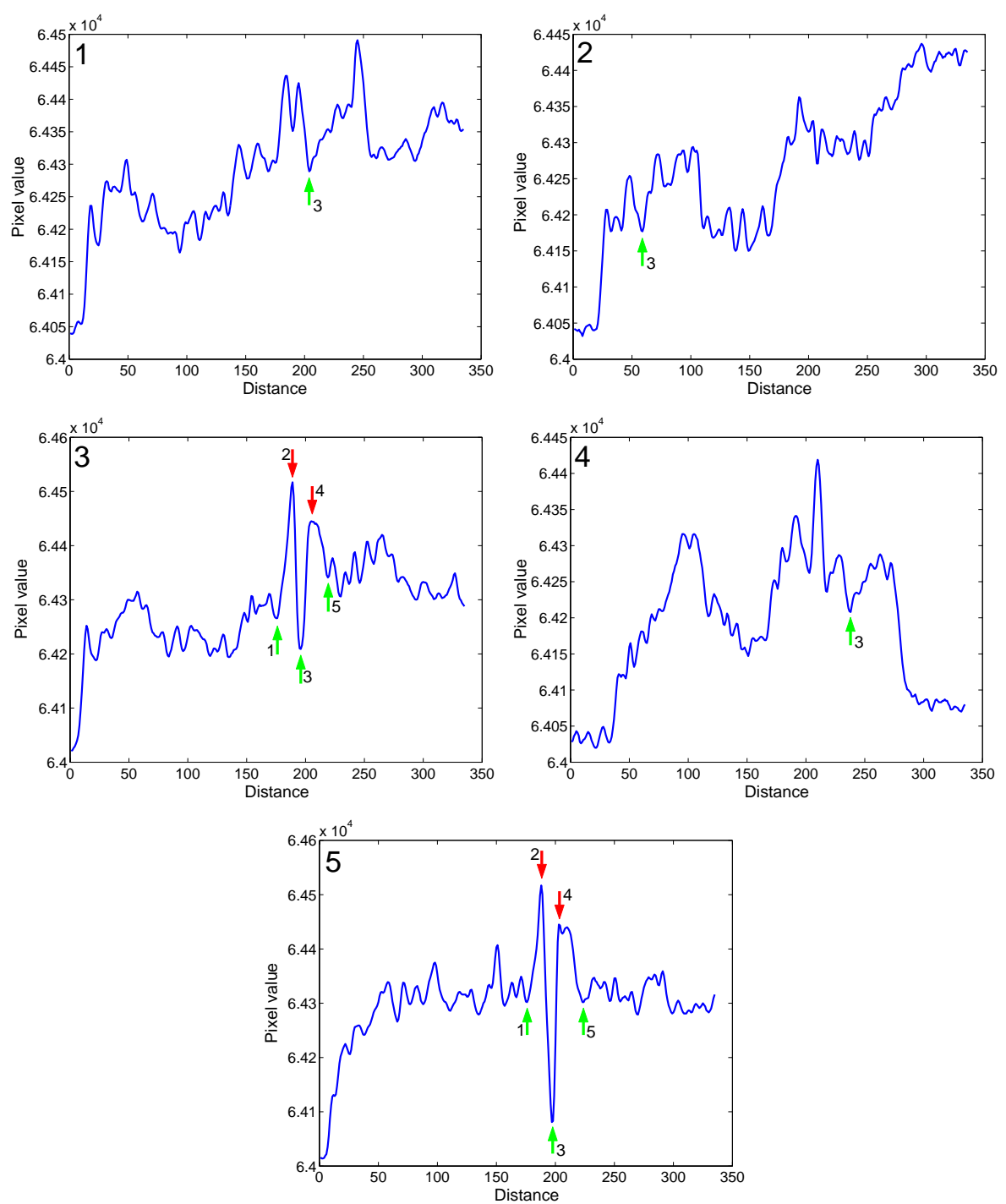

Fig. 7. Profile plots of line segments from Fig. 6 
tions (2nd line segment in Fig. 6). Another abnormal analysis may be made when a line segment goes through an area where two bones overlap (1st line segment). To prevent such mistakes, there are five or more, parallel line segments instead of one. Initially, each line segment is used to create its own profile plot. Afterwards, each profile plot is analyzed with the new formula which produces five pairs. Each pair contains $\mathrm{n}$ argument corresponding to a joint location point and function value for this feature. Finally, an accurate argument is chosen from the five of them in the following way.

Denote a set of five obtained pairs as $\left\{\left(x_{\text {joint }}^{p}, v_{\text {joint }}^{p}\right): p \in\{1, \ldots, 5\}\right\}$. Let $\min \in\{1, \ldots, 5\}$ be the index of the smallest argument and let $\max \in\{1, \ldots, 5\}$ be an index of the biggest argument from the set of pairs. Then an accurate pair index is given by the formula:

$$
a c c:=\widehat{p}: v_{\text {joint }}^{\widehat{p}}=\arg \max _{p \neq \min \wedge p \neq \max }\left[v_{\text {joint }}^{p}\right] .
$$

\section{Experiments}

In total, 1120 joints were located in 80 images (from 40 subjects) included in the test set, acquired through the offices of the Dietla Hospital in Cracow, Poland and the University Hospital in Cracow, Poland. The tested images presented bones with pathological changes or uninfected bones. The performance was assessed by measuring the percentage error of the two most frequently erroneous steps: thresholding and joint space location.

The thresholding percentage error (TPE) was calculated through a simple comparison of the number of inaccurately binarized images and the total number of images:

$$
\mathrm{TPE}=\frac{\text { inaccurate binarized }}{\text { all images }} * 100 \%
$$

TPE improved over the result in the previous article (from $5 \%$ to $\mathbf{0 \%}$ ).

The mean percentage errors of the second phase, the identification of the joint space locations, are given in Tab. 1 . The joint space location percentage error (JSLPE) was measured for every analyzed image by dividing the number of inaccurately located joints by 14 (the number of hand joints). Then, the mean of JSLPE and standard deviation of JSLPE was computed. Additionally, failure rates were calculated for each joint. 
Tab. 1. Mean joint space location percentage error in previous article and in current paper

\begin{tabular}{|l|r|r|}
\hline Joint name & Previous mean JSLPE & Improved mean JSLPE \\
\hline \hline Thumb - PIP & $19.74 \%$ & $3.75 \%$ \\
\hline Thumb - MCP & $14.47 \%$ & $6.25 \%$ \\
\hline Index finger - DIP & $2.63 \%$ & $1.25 \%$ \\
\hline Index finger - PIP & $6.58 \%$ & $0 \%$ \\
\hline Index finger - MCP & $3.95 \%$ & $0 \%$ \\
\hline Middle finger - DIP & $0 \%$ & $2.5 \%$ \\
\hline Middle finger - PIP & $2.63 \%$ & $0 \%$ \\
\hline Middle finger - MCP & $0 \%$ & $0 \%$ \\
\hline Ring finger - DIP & $0 \%$ & $2.5 \%$ \\
\hline Ring finger - PIP & $5.26 \%$ & $1.25 \%$ \\
\hline Ring finger - MCP & $0 \%$ & $0 \%$ \\
\hline Little finger - DIP & $9.21 \%$ & $2.5 \%$ \\
\hline Little finger - PIP & $6.58 \%$ & $3.5 \%$ \\
\hline Little finger - MCP & $14.47 \%$ & $3.75 \%$ \\
\hline GLOBAL - MCP & $6.11 \%+/-0.67 \%$ & $2.59 \%+/-4.72 \%$ \\
\hline
\end{tabular}

The JSLPE is $\mathbf{2 . 5 9 \%}+/ \mathbf{- 4 . 7 2 \%}$ and is much smaller than JSLPE in the previous paper $(6.11 \%+/-0.67 \%)$. It appears that the thumbs IP and MCP, little fingers DIP and little fingers MCP joints are located more often than before our improvements. The PIP joints are also located more frequently. On the other hand, surprisingly, the analysis of little finger PIP joints fails twice as often. This problem will become a topic for future research.

Finally, the overall percentage error (OPE), computed from the two errors mentioned above narrowed significantly from $10.8 \%$ to $\mathbf{2 . 5 9 \%}$ (Tab. 2).

\section{Discussion}

This paper describes an improvement of a method, already introduced in previous article, to quantify the minimal JSW in hand radiographs. The innovations affect two stages of the algorithm (thresholding and joint space location), but have an immeasurable, positive impact on the whole analysis.

The changes described in this paper reduce TPE from $5 \%$ to $0 \%$ and 
Tab. 2. Errors for the two analyzed steps and overall errors in previous article and in current paper

\begin{tabular}{|l|c|c|c|}
\hline & TPE & JSLPE & OPE \\
\hline \hline Previous error & $5 \%$ & $6.11 \%$ & $10.8 \%$ \\
\hline Previous error bar & & $1.54 \%$ & \\
\hline Improved error & $0 \%$ & $2.59 \%$ & $2.59 \%$ \\
\hline Improved error bar & & $4.72 \%$ & \\
\hline
\end{tabular}

JSLPE from $6.11 \%+/-1.54 \%$ to $2.59 \%+/-4.72 \%$, consequently decreasing OPE to $2.59 \%$. The difference in comparison with the previous error is $8.21 \%$, so improvement is significant. Computer interpretation of hand Xray images is extremely difficult due to the complexity of hand structure. In a few pioneering studies on this topic $[3,4,10,21,22,16,17,19]$, the results are far from satisfactory, semi-automated or incomplete. It seems that the results described in $[2,23]$ and the enhancement described in the present paper significantly improve those mentioned above.

In future studies, the author will attempt to apply the shape feature description language to the pattern of the upper and lower surface of joint space to pick up the abnormalities in the hand. Creating an algorithm for such a diagnosis seems to be an interesting avenue for future research.

\section{References}

[1] Bachmann D., Resnick D.; Radiological Atlas of Rheumatoid Diseases, Editiones Roche, Switzerland, 1994.

[2] Bielecki A., Korkosz M., Zieliński B.; Hand radiographs preprocessing, image representation in the finger regions and joint space width measurements for image interpretation, Pattern Recognition, 41(12), 2008, pp. 3786-3798.

[3] Beier D., Lehmann T.M., Seidl T., Thies C.; Segmentation of medical images combining local, regional, global, and hierarchical distances into a bottom-up region merging scheme, Proceedings of the SPIE, 5747, 2005, pp. 546-555.

[4] Botcher J. et al.; Computerized Digital Imaging Techniques Provided by Digital X-ray Radiogrammetry as New Diagnostic Tool in Rheumatoid Arthritis, Journal of Digital Imaging, 19(3), 2006, pp. 279-288. 
[5] Bublinski Z., Mikrut Z., Popiela-Mizera A.; A method of linear star section applied for object separation in ERCP images, International Conference of Image Processing, 1, 1996, pp. 363-366.

[6] Bublinski Z., Mikrut Z., Popiela-Mizera A., Zajac A.; A dedicated algorithm for object identification in medical images, Image Processing Communication, 1, 1995, pp. 43-58.

[7] Cooper C., Klippel J., Dieppe P.; Osteoarthritis and Related Disorders in Rheumatology, Mosby International, London, 1998.

[8] Freeman H.; On the encoding of arbitrary geometric configurations, IRE Transaction Electronical Computations, 10, 1961, pp. 260-268.

[9] Freeman H.; On the digital-computer classification of geometric line patterns, in Proceedings of the 18th National Electronics Conference, 1962, pp. $312-324$.

[10] van Kloster R. et al.; Automatic quantification of osteoarthritis in hand radiographs: validation of a new method to measure joint space width, Osteoarthritis and Cartilage, 16(1), 2008, pp. 18-25.

[11] Liang M., Esdaile J., Klippel J., Dieppe P.; Impact and Cost Effectiveness of Rheumatologic Care in Rheumatology, Mosby International, London 1998.

[12] MacGregor A., Silman A., Klippel J., Dieppe P.; Rheumatoid Arthritis in Rheumatology, Mosby International, London 1998.

[13] Otsu N.; A threshold selection method from gray-level histograms, IEEE Transaction Systems, Man, and Cybernetics, 9, 1979, pp. 62-66.

[14] Pavlidis T.; Algorithms for Graphics and Image Processing, Springer and Rockville Computer Science Press, Berlin 1982.

[15] Peterfy C., Klippel J., Dieppe P.; Imaging Techniques in Rheumatology, Mosby International, London 1998.

[16] Pfeil A. et al.; Normative Reference Values of Joint Space Width Estimated by Computer-aided Joint Space Analysis (CAJSA): The Distal Interphalangeal Joint, Journal of Digital Imaging, Suppl. 1, 2008, pp. 104-112.

[17] Pfeil A. et al.; Computer-aided joint space analysis of the metacarpalphalangeal and proximal-interphalangeal finger joint: normative age-related and gender-specific data, Skeletal Radiology, 36(9), 2007, pp. 853-864.

[18] Pratt W.K.; Digital Image Processing, John Wiley \& Sons, New York, 1991.

[19] Sharp J., Gardner J., Bennett E.; Computer-based methods for measuring joint space and estimating erosion volume in the finger and wrist joints of patients with rheumatoid arthritis, Arthritis \& Rheumatism, 43(6), 2000, pp. 1378-1386. 
[20] Szczeklik A., Zimmermann-Górska I.; Internal Medicine (Choroby wewnętrzne), Medycyna Praktyczna, Warszawa 2006 (in Polish).

[21] Tadeusiewicz R., Ogiela M.; Picture languages in automatic radiological palm interpretation, International Journal of Applied Mathematics and Computer Science, 15(2), 2005, pp. 305-312.

[22] Tadeusiewicz R., Ogiela M., Ogiela L.; Image languages in intelligent radiological palm diagnostics, Pattern Recognition, 39, 2006, pp. 2157-2165.

[23] Zieliński B.; Fully-automated algorithm dedicated to computing metacarpophalangeal and interphalangeal joint cavity widths, Schedae Informaticae, 16, 2007, pp. 47-67.

Received April 23, 2008 